This is an electronic reprint of the original article. This reprint may differ from the original in pagination and typographic detail. Author(s): Tero, Tiia-Riikka; Suhonen, Aku; Salorinne, Kirsi; Campos-Barbosa, Hélène; Nissinen,
Maija

Title: $\quad$ The Missing Member of the Partially O-Alkylated Resorcinarene Family: Synthesis and Conformation of Methyl Tetramethoxy Resorcinarene

Year: $\quad 2013$

Version:

Please cite the original version:

Tero, T.-R., Suhonen, A., Salorinne, K., Campos-Barbosa, H., \& Nissinen, M. (2013). The Missing Member of the Partially O-Alkylated Resorcinarene Family: Synthesis and Conformation of Methyl Tetramethoxy Resorcinarene. Organic Letters, 15(5), 1096-1099. https://doi.org/10.1021/ol400118t

All material supplied via JYX is protected by copyright and other intellectual property rights, and duplication or sale of all or part of any of the repository collections is not permitted, except that material may be duplicated by you for your research use or educational purposes in electronic or print form. You must obtain permission for any other use. Electronic or print copies may not be offered, whether for sale or otherwise to anyone who is not an authorised user. 


\title{
The Missing Member of the Partially 0 -Alkylated Resorcinarene Family: Synthesis and Conformation of Methyl Tetramethoxy Resorcinarene
}

\author{
Tiia-Riikka Tero, Aku Suhonen, Kirsi Salorinne, Hélène Campos- \\ Barbosa $^{\dagger}$ and Maija Nissinen*
}

Nanoscience Center, Department of Chemistry, University of Jyväskylä, P.O. Box 35, FIN-40014 JYU, Finland

maija.nissinen@jyu.fi

Received Date (will be automatically inserted after manuscript is accepted)

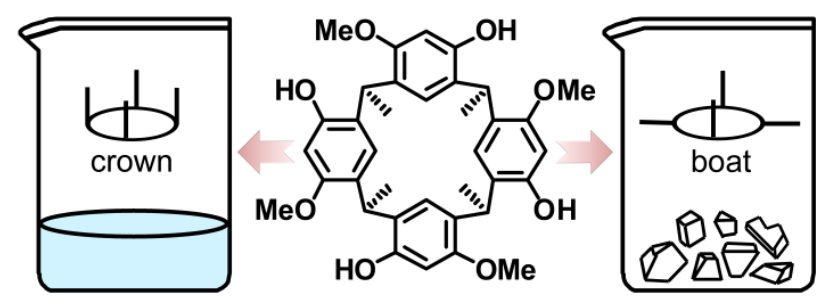

An improved Lewis acid catalyzed synthesis method for methyl tetramethoxy resorcinarene is described, which produced the missing lower rim methyl derivative of this partially 0 -alkylated resorcinarene family. Structural characterization by means of variable temperature NMR experiments and single crystal X-ray diffraction studies furthermore revealed that the resorcinarene core adopts different conformations in the solid state and in solution.

Partially $O$-alkylated resorcinarene derivatives are particularly interesting due to their diverse possibilities in functionalization and potential for the preparation of enantiomerically pure chiral resorcinarene derivatives. ${ }^{1}$ In 2000 McIldowie et al. ${ }^{2}$ described a Lewis acid catalyzed synthesis of inherently chiral $C_{4}$ symmetric tetramethoxy resorcinarenes - a breakthrough in obtaining partially $O$ alkylated resorcinarenes in a convenient way. These derivatives have been advantageous platforms for example in the construction of Fréchet-type resorcinarene

$\dagger$ Current address: Ecole Nationale Supérieure de Chimie de Mulhouse, France.

(1) For a review see: Moore, D.; Matthews, S. E. J. Inclusion Phenom. Macrocyclic Chem. 2009, 65, 137.

(2) McIldowie, M. J.; Morcerino, M.; Skelton, B. W.; White, A. H. Org. Lett. 2000, 2, 3869. CCDC 151370. dendrimers, ${ }^{3}$ resorcinarene mono- and bis-crowns ${ }^{4}$ and resorcinarene tetrapodands ${ }^{5}$, some of which have been shown to coordinate anions, alkaline- and transition metal

(3) Luostarinen, M.; Salorinne, K.; Lähteenmäki, H.; Mansikkamäki, H.; Schalley, C. A.; Nissinen, M.; Rissanen, K. J. Inclusion Phenom. Macrocyclic Chem. 2007, 58, 71. CCDC 256240, 256241.

(4) (a) Salorinne, K.; Nissinen, M. Org. Lett. 2006, 8, 5473. (b) Salorinne, K.; Nissinen, M. Tetrahedron 2008, 64, 1798. (c) Salorinne, K.; Lopez-Acevedo, O.; Nauha, E.; Häkkinen, H.; Nissinen, M. CrystEngComm 2012, 14, 347. (d) Salorinne, K.; Nauha, E.; Nissinen, M. Chem. Asian J. 2012, 7, 809. (e) Salorinne, K.; Tero, T.-R.; Riikonen, K.; Nissinen, M. Org. Biomol. Chem. 2009, 7, 4211.

(5) See for example (a) Salorinne, K.; Weimann, D. P.; Schalley, C. A.; Nissinen, M. Eur. J. Org. Chem. 2009, 6151. (b) Mclldowie, M. J.; Morcerino, M.; Ogden, M. I.; Skelton, B. W. Tetrahedron 2008, 64 1798. (c) Tero, T.-R.; Salorinne, K.; Nissinen, M. CrystEngComm 2012, 14, 7360. (d) Klaes, M.; Neumann, B.; Stammler, H.-G.; Mattay, J. Eur. J. Org. Chem. 2005, 864. (e) Bulman Page, P. C.; Chan, Y.; Heaney, H. McGrath, M. J.; Moreno, E. Eur. J. Org. Chem. 2011, 5347.(f) Wiegmann, S.; Neumann, B.; Stammler, H.-G.; Mattay, J. Eur. J. Org. Chem. 2012, 3955. 
cations, and ammonium guests. The partial $O$-methylated resorcinarenes have also enabled the synthesis of other aromatic ring functionalized resorcinarene derivatives leading to tetrabiaryl cavity-extended resorcinarenes and resorcinarene tetrabenzoxazines. ${ }^{6}$ Despite all the research done with tetramethoxy resorcinarenes to date, having the lower rim alkyl chain length varying from two carbon atoms (C2) up to eleven carbon atoms (C11), the tetramethoxy resorcinarene family has lacked an efficient synthesis method for its smallest member - the one with methyl group $(\mathrm{C} 1)$ at the lower rim. ${ }^{7}$

The $C$-methyl resorcinarenes have been shown to be conformationally remarkably flexible adopting at least crown, boat, chair and scoop conformations in the solid state. ${ }^{8}$ Both the adaptable scaffold and the free hydroxyl groups have enabled unique formations of various supramolecular assemblies, nanotubes and capsules. ${ }^{9}$ This encouraged us to look for a synthesis strategy for the preparation of C1-tetramethoxy resorcinarene - not only being interesting structure on its own but also to be used as a platform for further functionalizations. Herein, we report a successful synthesis method for the preparation of C1-tetramethoxy resorcinarene (1). In addition, variable temperature (VT) NMR spectroscopy and single crystal X-ray diffraction studies were used to investigate the structural characteristics that result from the short methyl group at the lower rim.

The standard protocol for the preparation of tetra $O$ methylated resorcinarenes reported by McIldowie et al. ${ }^{2}$ describes a one-pot reaction of an aldehyde ${ }^{10}$ with 3methoxy phenol in the presence of a Lewis acid $\mathrm{BF}_{3} \cdot \mathrm{Et}_{2} \mathrm{O}$ in dry dichloromethane at room temperature (Scheme 1). This protocol produces the C2- to C11-tetramethoxy resorcinarenes with good to excellent yields. However, when the reaction was repeated with acetaldehyde in order to produce C1-tetramethoxy resorcinarene, an unidentified polymeric product was obtained (Table 1, Entry 1). ${ }^{11}$

Intrigued by this we set out to test whether different reaction conditions would favor the production of the

(6) (a) Paletta, M.; Klaes, M.; Neumann, B.; Stammler, H.-G.; Grimme, S.; Mattay, J. Eur. J. Org. Chem. 2008, 555. (b) Buckley, B. R.; Boxhall, J. Y.; Bulman Page, P. C.; Chan, Y.; Elsegood, M. R. J.; Heaney, H.; Holmes, K. E.; Mclldowie, M. J.; McKee, V.; McGrath, M. J.; Morcerino, M.; Poulton, A. M.; Sampler, E. P.; Skelton, B. W.; White, A. H. Eur. J. Org. Chem. 2006, 5117.

(7) We have found a Japanese patent mentioning the structure of C1tetramethoxy resorcinarene in an application for heat-resistant flat films. However, no details of the synthesis appear to be reported. Momota, J.; Onishi, H. Jpn. Kokai Tokkyo Koho, JP 2004018421 A 20040122, 2004.

(8) (a) MacGillvray, L. R.; Diamente, P. R.; Reid, J. L.; Ripmeester, J. A. Chem. Commun. 2000, 359. (b) Zhang, Y.; Kim, C. D.; Coppens, P. Chem. Commun. 2000, 2299. (c) Ma, B.-Q.; Zhang, Y.; Coppens, P. CrystEngComm 2001, 3, 78. (d) Ma, B.-Q.; Coppens, P. Chem. Commun. 2002, 424.

(9) See for example (a) Ma, B.-Q.; Coppens, P. Cryst. Growth Des. 2007, 4, 1377. (b) Mansikkamäki, H.; Nissinen, M.; Rissanen, R. Angew. Chem. Int. Ed. 2004, 43, 1243. (c) Matheny, J. M.; Bosch, E. Cryst. Growth Des. 2007, 7, 984. (d) MacGillivray, L. R.; Atwood, J. L. Nature 1997, 386, 469.

(10) Aldehydes ranging from propanal to dodecanal have been successful by the standard protocol.

(11) Klaes, M.; Agena, C.; Köhler, M.; Inoue, M.; Wada, T.; Inoue, Y.; Mattay, J. Eur. J. Org. Chem. 2003, 1404.
Scheme 1. Synthesis of C1-tetramethoxy resorcinarene (1) with crystallographic numbering shown for the core. Schematic presentations for the boat and the crown conformations are shown below.<smiles>CCOCCCCC(=O)[O+]=O</smiles>

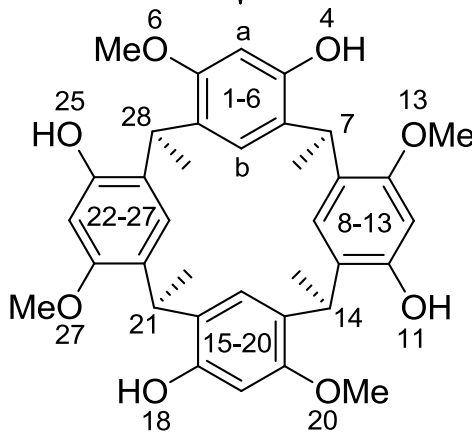

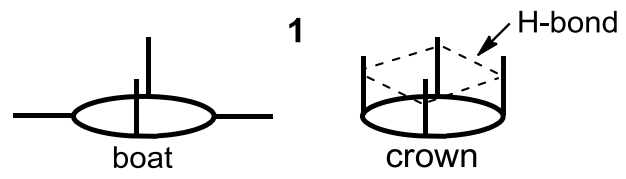

Table 1. The optimized reaction conditions for the synthesis of macrocycle 1 .

\begin{tabular}{lllll}
\hline Entry & $\mathrm{c}(\mathrm{mol} / \mathrm{l})^{a}$ & $t^{b}\left({ }^{\circ} \mathrm{C}\right)$ & $t^{c}\left({ }^{\circ} \mathrm{C}\right)$ & Product (yield) \\
\hline 1 & $1.64 / 1.72$ & $\sim 21$ & reflux & polymer \\
2 & $0.21 / 0.27$ & 21 & $<30$ & macrocycle $\mathbf{1}+$ polymer \\
3 & $1.64 / 1.72$ & 1 & $<20$ & macrocycle $\mathbf{1}(33 \%)$ \\
4 & $1.64 / 1.72$ & -40 & -20 & macrocycle $\mathbf{1}(18 \%)$ \\
\multicolumn{4}{c}{${ }^{a} \mathrm{Concentrations} \mathrm{of}^{2}$-methoxyphenol/acetaldehyde in the reaction } \\
mixture. ${ }^{b}$ Temperature of the reaction mixture before the addition of \\
$\mathrm{BF}_{3} \cdot \mathrm{Et}_{2} \mathrm{O}$. ${ }^{c}$ Temperature of the reaction mixture during the addition of \\
$\mathrm{BF}_{3} \cdot \mathrm{Et}_{2} \mathrm{O}$.
\end{tabular}

macrocycle 1 over the polymeric product obtained under the standard reaction conditions (Table 1). First, a high dilution conditions were used resulting in a mixture that contained both the macrocycle $\mathbf{1}$ and the polymeric product (Entry 2). The foremost difference to the standard protocol was that the reaction was less exothermic as the temperature rose at its highest to $30^{\circ} \mathrm{C}$, whereas in the standard protocol a higher increase in temperature (> $40^{\circ} \mathrm{C}$ ) was observed. This indicated that for $\mathrm{C} 1$ tetramethoxy resorcinarene a slower reaction rate might be more optimal for the macrocycle formation thus hinting to be a kinetic reaction whereas the formation of a polymer being a thermodynamic reaction. The reaction was repeated with standard concentration at lower 
temperatures in an ice bath and at explicitly lower temperature of $-40{ }^{\circ} \mathrm{C}$ to slow down the reaction (entries 3 and 4). Both of these experiments produced the pure macrocycle 1 with moderate yields of $18-33 \%$. These results indicate that the temperature of the reaction mixture has a more pronounced role in obtaining the pure macrocycle 1 than the high-dilution conditions.

The ${ }^{1} \mathrm{H}$ NMR spectra of macrocycle $\mathbf{1}$ at room temperature shows one set of resonances to the protons of the resorcinarene core, which is indicative of a $\mathrm{C}_{4}$ symmetric crown conformation (Figure $1,30^{\circ} \mathrm{C}$ ). ${ }^{2}$

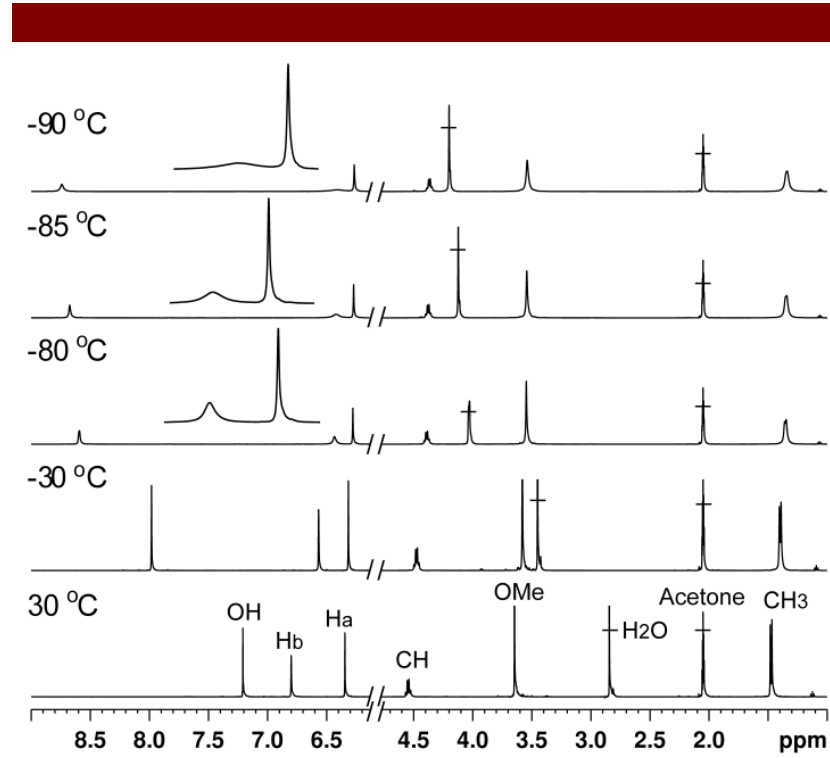

Figure 1. ${ }^{1} \mathrm{H}$ NMR variable temperature experiment of compound 1 in acetone- $d_{6}$ showing the spectra measured at a temperature range of 30 to $-90{ }^{\circ} \mathrm{C}$. A close-up of the region 6.30-6.60 ppm is shown for the spectra of -80 to $-90{ }^{\circ} \mathrm{C}$.

However, the resorcinarene core could also adopt less symmetric conformations giving rise to multiple signals of the resorcinarene core, but due to fast interconversion between the conformations on the NMR time scale this might not be detected at room temperature. ${ }^{12}$ Therefore, VT NMR measurements were undertaken in a non-polar $\mathrm{CDCl}_{3}$ solvent and in a polar acetone- $d_{6}$ solvent at a temperature range of 30 to -60 or $-90^{\circ} \mathrm{C}$, respectively, in order to confirm the conformation of macrocycle $\mathbf{1}$ in solution. Even at remarkably low temperature of $-60{ }^{\circ} \mathrm{C}$ in the non-polar $\mathrm{CDCl}_{3}$ solvent all the signals remained sharp (Figure S1). This observation indicated that no boat-to-boat interconversion or other less symmetric conformations occurred as no extensive broadening or splitting of the signals were observed and confirming the crown conformation of macrocycle 1 . This was expected since macrocycle 1 cannot form intermolecular hydrogen bonds with the non-polar $\mathrm{CDCl}_{3}$ solvent. Instead, macrocycle 1 forms intramolecular hydrogen bonding

(12) Abis, L.; Dalcanale, E.; Du vosel, A.; Spera, S. J. Chem. Soc. Perkin Trans. 2 1990, 2075. network on the upper ring stabilizing to the crown conformation. ${ }^{13}$ The only significant difference observed in the spectra measured at different temperatures was the downfield shift of the hydroxyl protons by $0.40 \mathrm{ppm}$, which was due to the $\mathrm{OH}$ signal shifting upon the change in temperature. $^{14}$

The VT NMR spectra measured in the polar acetone- $d_{6}$ also indicated a crown conformation. Even though some signals broadened at lower temperatures, no splitting occurred (Figure 1). All but the $\mathrm{OH}$-proton signal of macrocycle 1 was observed to shift somewhat upfield by $\left(0.08 / \mathrm{H}_{\mathrm{a}}-0.38 / \mathrm{H}_{\mathrm{b}} \mathrm{ppm}\right)$. The signal of the $\mathrm{OH}$ group on the other hand had a substantial downfield shift of 1.53 ppm and, in addition, the residual water signal in the solution was observed to have a similar downfield shift of $1.36 \mathrm{ppm}$. It is likely that the methoxy and hydroxyl groups at the upper rim interact with the residual water and acetone- $d_{6}$, but intermolecular hydrogen bonds do not seem to break the intramolecular hydrogen bonding network of macrocycle 1 , which ultimately keeps the macrocycle in the crown conformation.

The structural properties of $\mathbf{1}$ were also studied in the solid state by XRD. Single crystals of compound 1 suitable for crystal structure analysis were grown by vapor diffusion (solute-antisolvent) from ethyl acetatehexane (structure I) and methanol-water (structure II). Structure I crystallized in a monoclinic space group $P 2_{1} / n$ as a 1:1 water solvate. Structure II was obtained in a triclinic space group $P-1$ having one methanol and one water molecule per compound 1. In both structures I-II the resorcinarene core is in a boat conformation, despite the capacity of the upper rim to form intramolecular hydrogen bonds stabilizing the core into a crown conformation (Figure S2). ${ }^{15}$ All previously reported structures of tetramethoxy resorcinarenes with varying alkyl chain lengths have the resorcinarene cores more or less in the hydrogen bond stabilized crown conformation. ${ }^{2,3,15,16}$ However, boat conformation is not uncommon with the solid state structures of octahydroxyl $C$-methyl resorcinarenes. ${ }^{8 \mathrm{~b}, 17}$ In those reported structures the boat conformations are generally in a "brick" like rows stabilized by intermolecular hydrogen bonds and $\pi-\pi$ interactions between the resorcinarene molecules. The reason why the boat conformation is more common with the short alkyl chain resorcinarenes reasoned to be due to the ideal close packing compared to the longer alkyl chain resorcinarenes, which tend to crystallize into lipid-like bilayers with upper rim in a crown conformation. ${ }^{18}$

(13) Schiel, C.; Hembury, G. A.; Borovkov, V. V.; Klaes, M.; Agena, C.; Wada, T.; Grimme, S.; Inoue, Y.; Mattay, J. J. Org. Chem. 2006, 71 976.

(14) Friebolin, H. Basic One- and Two-Dimensional NMR spectroscopy, 3rd ed.; Whiley-VCH: Wheinheim, Germany, 1998.

(15) Salorinne, K.; Nissinen, M. CrystEngComm 2009, 11, 1572. CCDC 718573-718575.

(16) Busi, S.; Saxell, H.; Fröhlich, R.; Rissanen, K. CrystEngComm 2008, 10, 1803. CCDC 690122.

(17) Åhman, A.; Nissinen, M. Chem. Commun. 2006, 1209

(18) (a) Kuzmicz, R.; Dobrzycki, L.; Wosniak, K.; Benevelli, F.; Klinowski, J.; Kolodziejski, W. Phys. Chem. Chem. Phys. 2002, 4, 2387. (b) Mansikkamäki, H.; Schalley, C. A.; Nissinen, M.; Rissanen, 
In the case of macrocycle $\mathbf{1}$, the crystal packing is likewise clearly affected by the intermolecular hydrogen bonds formed in the crystal lattice, which also explain the preferred boat conformation of macrocycle $\mathbf{1}$ in the solid state. In structure I a bridging water molecule connects two same handed adjacent resorcinarene enantiomers 1 (A and $\mathrm{B}$ ) together via three intermolecular hydrogen bonds (2.03-2.38 $\AA$, Figure 2) by having two differerent

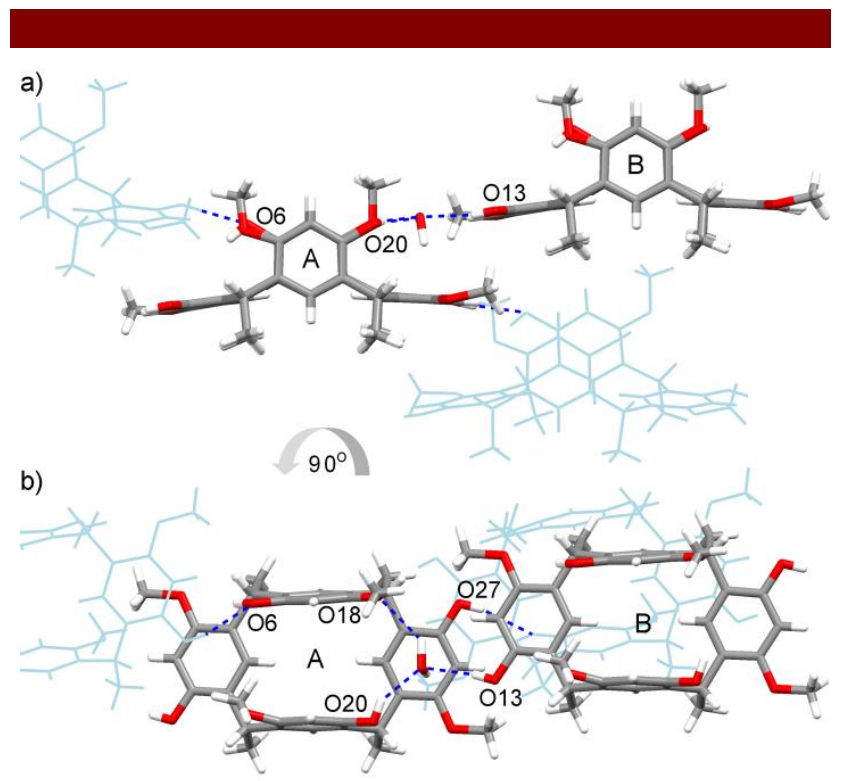

Figure 2. Hydrogen bonding network of structure $\mathbf{I}$ of macrocycle 1, shown from side (a) and top views (b). Resorcinares $\mathrm{A}$ and $\mathrm{B}$ joined together via water molecule are presented with elemental colors and acceptor/donor of direct hydrogen bonds between resorcinarenes with light blue. The hydrogen bonds are shown as blue dotted lines.

roles acting both as a hydrogen bond donor and an acceptor. Water molecule as the acceptor atom, intermolecular hydrogen bonds are formed with the hydroxyl groups of the two adjacent resorcinarenes (O20/upright-A and O13/horizontal-B), while the other role of the water atom as the donor atom leads to formation of hydrogen bonds to the resorcinarene methoxy group (O18). Two additional direct intermolecular hydrogen bonds $(2.22 \AA)$ are formed via the remaining hydroxyl groups of each resorcinarene (O6/upright-A, and O27/horizontal-A) with the respective hydroxyl groups of two neighboring different handed resorcinarene molecules (Figure 2).

Unlike in structure I, in structure II a three-moleculeunit is formed by one water molecule connecting three neighboring resorcinarene molecules $\mathbf{1}(\mathrm{A}, \mathrm{B}$ and $\mathrm{C}$ ) via three hydrogen bonds to two horizontal (O13-A and O27C) and to one upright hydroxyl (O6-B) groups (1.98-2.08 $\AA$, Figure 3). A horizontal methoxy group oxygen (O25-

K. New J. Chem. 2005, 29, 116. (c) Momose, A. A.; Bosch, E. Cryst. Growth Des. 2010, 10, 4043.

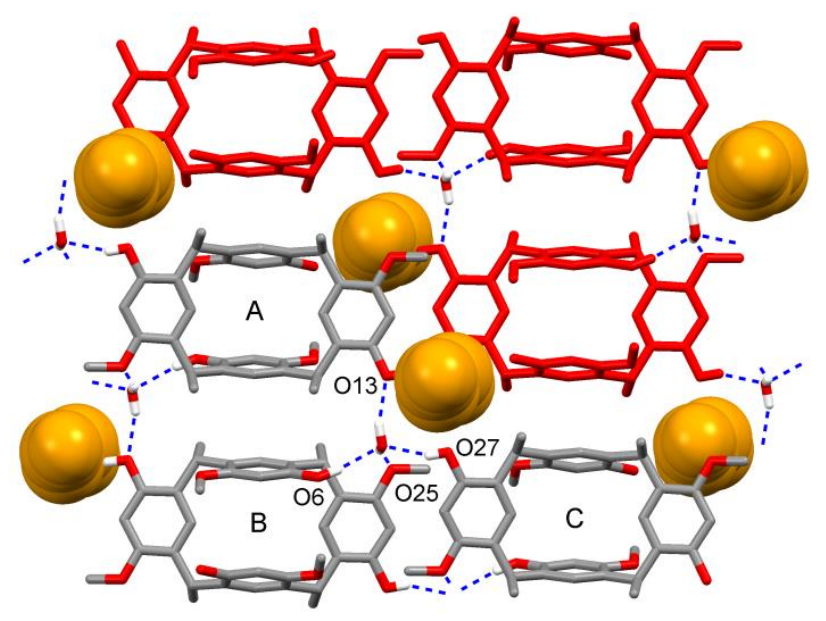

Figure 3. Hydrogen bonding network of structure II showing solvent methanol as a space fill model. The adjacent threeresorcinarene-unit is shown in red and the resorcinarenes connected with water molecule are labeled A-C. Noncoordinating hydrogens have been omitted for clarity and hydrogen bonds are shown as blue dotted lines.

B) of the resorcinarene $\mathrm{B}$ in the unit acts also as an acceptor for the fourth intermolecular hydrogen bond formed by the bridging water molecule $(2.00 \AA)$. Solvent methanol molecules fill up the remaining voids between the two adjacent three-resorcinarene-assemblies. The repeating three-molecule-units form layer like structures, which are piled on top of each other in the crystal lattice.

Even with different kinds of hydrogen bond networks both structures I and II form similar packing style. The chains (structure I, Figure S3) are positioned over each other forming a slightly oblique "brick wall" while the layers (structure II, Figure S4) are piled straight. The shortest possible alkyl chain on the lower rim enables this very close packing of resorcinarene $\mathbf{1}$ since methyl group requires only a small volume. Due to this unique structural characteristic of the $\mathrm{C} 1$ derivative, the lower rim alkyl chain does not seem to be the major contributor in the crystal packing as generally is the case with the longer alkyl chain derivatives. The major role therefore in the formation of crystal packing interactions falls down to the upper rim functionalities. This property together with the "brick" form of the macrocycle will enable various supramolecular assemblies (e.g. nanorod, nanotube and hexameric capsules) ${ }^{9}$ via further derivatizations and cocrystallizations that can be useful in making crystal engineered materials.

In conclusion, C1-tetramethoxy resorcinarene having methyl chains on its lower rim was successfully synthesized from 3-methoxyphenol and acetaldehyde by an improved synthesis method. The pure macrocycle was achieved at a reaction temperature below $0^{\circ} \mathrm{C}$ as opposed to the unidentified polymeric product obtained when the reaction was performed using standard reaction conditions at room temperature. VT NMR measurements showed that the resorcinarene core adapts a crown 
conformation both in polar and non-polar solvents. In contrary, in the crystal structures obtained the core adopts a boat conformation as the intramolecular hydrogen bonds of the upper rim are replaced with intermolecular interactions. These unique structural characteristics of the up-to-now missing C1-tetramethoxy resorcinarene will add new possibilities to the macrocyclic family of tetramethoxy resorcinarenes in terms of supramolecular self-assembly, crystal engineering and further functionalization.

Acknowledgment. We acknowledge Mr. Esa Haapaniemi ${ }^{\sharp}$ for his help with the VT NMR experiment and Dr. Kaisa Helttunen for her help with a measurement of one of the crystal structures. Financial support from the National Doctoral Programme in Nanoscience (NGSNano) is greatly appreciated.

Supporting Information Available. Experimental procedures and characterization for compound 1; two additional structures III and IV; additional figures of XRD structures and a scatter plot presentation of the results of CCDC structure search; crystal data and CIF for all structures. This material is available free of charge via the Internet at http://pubs.acs.org. 